\title{
Does Fourier analysis yield reliable amplitudes of quantum oscillations?
}

\author{
Alain Audouard ${ }^{1}$ and Jean-Yves Fortin ${ }^{2, *}$ \\ ${ }^{1}$ Laboratoire National des Champs Magnétiques Intenses, UPR 3228 CNRS, INSA, UGA, UPS, 143 avenue de Rangueil, \\ 31400 Toulouse, France \\ ${ }^{2}$ Laboratoire de Physique et Chimie Théoriques, CNRS UMR7019, Université de Lorraine, Vandoeuvre-lès-Nancy 54506, France
}

Received: 4 December 2017 / Received in final form: 6 July 2018 / Accepted: 17 July 2018

\begin{abstract}
Quantum oscillation amplitudes of multiband metals, such as high- $\mathrm{T}_{c}$ superconductors in the normal state, heavy fermions or organic conductors, are generally determined through Fourier analysis of the data even though the oscillatory part of the signal is field-dependent. It is demonstrated that the amplitude of a given Fourier component can strongly depend on both the nature of the windowing (either flat, Hahn or Blackman window) and, since oscillations are obtained within a finite field range, the window width. Consequences on the determination of the Fourier amplitudes, hence of the effective masses, are examined in order to determine the conditions for reliable data analysis.
\end{abstract}

\section{Introduction}

Quantum oscillations, the extrema of which are periodic in inverse magnetic field, are known to provide valuable information for the study of Fermi surface of metals. In particular, in addition to their frequencies which are directly related to the closed orbit areas on the Fermi surface, field and temperature dependence of their amplitudes allows for the determination of the effective masses and scattering rates [1]. Multiband metals such as heavy fermions [2] or high- $\mathrm{T}_{c}$ superconducting iron chalcogenides [3-6] have a complex Fermi surface due to numerous sheets crossing the Fermi level, giving rise to many orbits under a magnetic field, hence to a complex quantum oscillation spectrum. Besides, in the case where magnetic breakdown (MB) or quantum tunneling of quasiparticles between orbits occurs, as in many organic metals $[7,8]$, additional orbits are further generated. In such cases, data can be readily derived through a Fourier analysis, allowing discrimination between the various frequencies. The point is that the amplitude of quantum oscillations is field dependent. Therefore, strictly speaking, they are not periodic in inverse field. The question that arises is then to determine to what extent reliable temperature- and fielddependent amplitudes can be derived from the Fourier analysis of such field-dependent data.

In the following, we consider the organic metal $\theta$-(ET) ${ }_{4} \mathrm{ZnBr}_{4}\left(\mathrm{C}_{6} \mathrm{H}_{4} \mathrm{Cl}_{2}\right)$, for which the de Haas-van Alphen (dHvA) and Shubnikov-de Haas (SdH) oscillations were extensively studied [9] in pulsed magnetic fields of up to $55 \mathrm{~T}$. As it is the case of many compounds based on the bis(ethylenedithio)tetrathiafulvalene molecule

\footnotetext{
* e-mail: jean-yves.fortin@univ-lorraine.fr
}

(abbreviated as ET), this compound illustrates the model Fermi surface proposed by Pippard to compute magnetic breakdown amplitudes of multiband metals [10]. As reported in reference [9] and depicted in Figure 1, its Fermi surface is composed of one strongly two-dimensional closed orbit $(\alpha)$ and a pair of quasi-one-dimensional sheets giving rise in a magnetic field to the orbit $\beta$ originated from MB. As a result, the oscillation spectrum is composed of many frequencies which are linear combinations of the frequencies linked to the $\alpha$ and $\beta$ orbits. Amplitudes relevant to these combinations are strongly influenced by oscillations of the chemical potential in the magnetic field $[9,11]$. Nevertheless, it appears that this phenomenon has negligible influence on the amplitude of the dominant components $\alpha$ and $\beta$, allowing relevant data analysis on the basis of the Lifshitz-Kosevich formalism [12].

Rather than bringing additional information on this compound, the aim of this paper is to determine to what extent the Fourier analysis is able to yield reliable values of physical parameters of interest, in particular effective masses and scattering rates (through the Dingle temperature). To this purpose, we will consider the $\beta$ orbit [9], with frequency $f_{0}=f_{\beta}=4534 \mathrm{~T}$, effective mass $m^{*}=m_{\beta}=3.4 m_{\mathrm{e}}$ and $T_{\mathrm{D}}=0.8 \mathrm{~K}$ (this latter parameter being dependent on the considered crystal), and which involves no reflections and four tunnelings with MB field $B_{0}=26 \mathrm{~T}$. This component will serve as a basis to determine the influence of the windowing (nature and width) on the Fourier amplitude evaluation.

\section{Methodology}

At a fixed temperature $T$, a given Fourier component of the oscillatory part of magnetization ( $\mathrm{dHvA}$ oscillations) and conductivity (SdH oscillations) can be generally written 


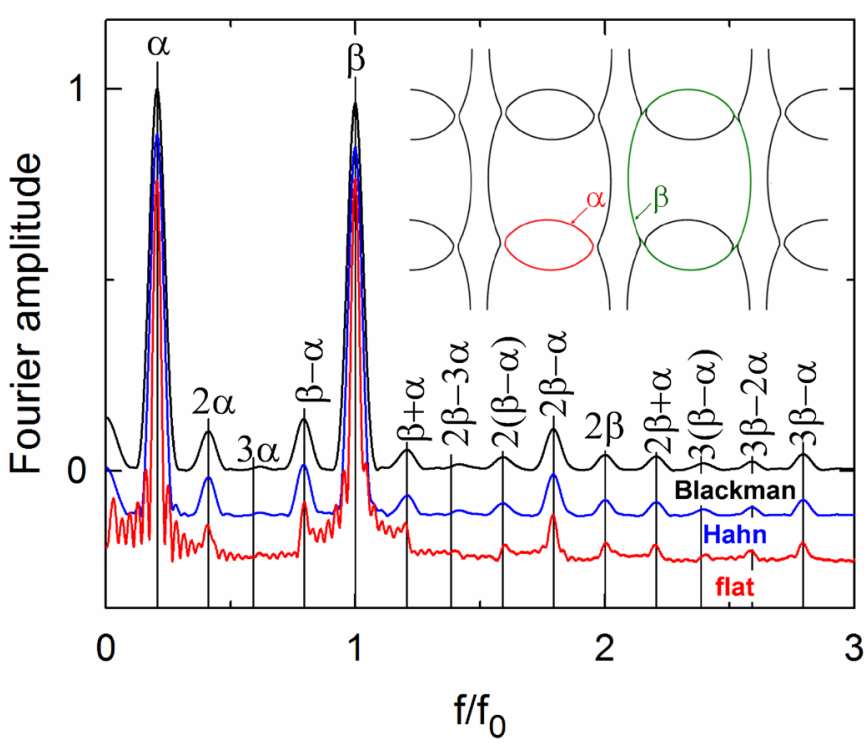

Fig. 1. Fourier analysis of de Haas-van Alphen oscillations of the organic metal $\theta$ - $(\mathrm{ET})_{4} \mathrm{ZnBr}_{4}\left(\mathrm{C}_{6} \mathrm{H}_{4} \mathrm{Cl}_{2}\right)$, obtained with Blackman, Hahn and flat windows in the field range 40-56 T at $2 \mathrm{~K}$, see text for details. Vertical lines are marks calculated with $f_{\alpha}=930 \mathrm{~T}$ and $f_{0}=f_{\beta}=4534 \mathrm{~T}$. The insert displays the Fermi surface in which the $\alpha$ and $\beta$ orbits are indicated (data are from Ref. [9]).

as $A(x)=A_{0}(x) \sin \left(2 \pi f_{0} x+\phi\right)$, where $x=1 / B, f_{0}$ is the frequency and $\phi$ is, for normal metals, the Onsager phase, generally equal to the product of $\pi$ and the harmonic number. In the framework of the Lifshitz-Kosevich and Falicov-Stachowiak formalism [1,12,13], the amplitude is given by a product of amplitude damping factors $A_{0}(x)$ $\propto R_{\mathrm{T}} R_{\mathrm{D}} R_{\mathrm{MB}}$ for a given field direction (in which case the spin damping factor is a field- and temperatureindependent prefactor). For a two-dimensional orbit, the thermal, Dingle and MB damping factors are given by the expressions $R_{\mathrm{T}}=u_{0} T m^{*} x / \sinh \left(u_{0} T m^{*} x\right), R_{\mathrm{D}}=\mathrm{e}^{-u_{0} T_{\mathrm{D}} m^{*} x}$ and $R_{\mathrm{MB}}=\mathrm{e}^{-n_{\mathrm{t}} B_{0} x / 2}\left(1-\mathrm{e}^{-B_{0} x}\right)^{n_{r} / 2}$, respectively, where $u_{0}=2 \pi^{2} k_{\mathrm{B}} m_{\mathrm{e}}(e \hbar)^{-1}=14.694 \mathrm{~T} / \mathrm{K}, m^{*}$ is the effective mass and $T_{\mathrm{D}}$ is the Dingle temperature $\left(T_{\mathrm{D}}=\hbar / 2 \pi k_{\mathrm{B}} \tau\right.$, where $\tau$ is the relaxation time). $n_{\mathrm{t}}$ and $n_{\mathrm{r}}$ are the number of tunneling and reflections the quasi-particles are facing during their travel along a MB orbit with a MB gap $B_{0}$. In the following, we will consider $\mathrm{dHvA}$ oscillations relevant to the above-mentioned $\beta$ orbit. Since measured magnetic torque $\tau$ is related to magnetization $\mathbf{M}$ as $\tau=\mathbf{M} \times \mathbf{B}$, the Fourier amplitude can be written as

$$
A_{0}(x) \propto \frac{T m_{\beta}}{\sinh \left(u_{0} T m_{\beta} x\right)} \exp \left[-\left(u_{0} T_{\mathrm{D}} m_{\beta}+2 B_{0}\right) x\right]
$$

At high-enough values of $u_{0} T m^{*} x, A_{0}(x)$ can be approximated as

$$
A_{0}(x) \simeq a_{0} \exp (-\lambda x)
$$

where $a_{0}$ is a temperature-dependent prefactor $\left(a_{0} \propto T\right)$ and $\lambda=u_{0}\left(T+T_{\mathrm{D}}\right) m_{\beta}+2 B_{0} .{ }^{1}$ This approximation provides a single parameter characterizing the field dependence of the amplitude: the largest the $\lambda$, the steepest the field dependence. For $\theta$-(ET) ${ }_{4} \mathrm{ZnBr}_{4}\left(\mathrm{C}_{6} \mathrm{H}_{4} \mathrm{Cl}_{2}\right)$, explored $\lambda$ values are within $194 \mathrm{~T}$ at $2 \mathrm{~K}$ and $305 \mathrm{~T}$ at $4.2 \mathrm{~K}$. Due to large Dingle temperature, even larger values are obtained for the high- $\mathrm{T}_{c}$ superconductor $\mathrm{FeSe}$, for which $\lambda$ varies from $250 \mathrm{~T}$ at $1.6 \mathrm{~K}$ to $370 \mathrm{~T}$ at $4.2 \mathrm{~K} \mathrm{[6]}$.

Since the signal amplitude is field-dependent, windowing $[14-18]$ is mandatory in order to determine the Fourier amplitude at a given inverse field value $\bar{x}$. The inverse field range $\Delta x$ is within $x_{m}$ and $x_{M}\left(\Delta x=x_{M}-x_{m}\right)$ and centered on $\bar{x}=\left(x_{m}+x_{M}\right) / 2$. In order to explore the influence of windowing on the Fourier amplitude, flat, Hahn and Blackman windows are considered in the following: $w(x)=1, w(x)=\{1+\cos [2 \pi(x-\bar{x}) / \Delta x]\} / 2$, and $w(x)=$ $0.42+0.5 \cos [2 \pi(x-\bar{x}) / \Delta x]+0.08 \cos [4 \pi(x-\bar{x}) / \Delta x]$, respectively, within the range $x_{m}$ to $x_{M}$ and $w(x)=0$ everywhere else. We can write more generally the window function as $w(x)=\sum_{n \geq 0}^{p} c_{n} \cos [2 \pi n(x-\bar{x}) / \Delta x]$, where $p=0,1,2$ for a flat, Hahn and Blackman window, respectively, but this can be generalized for higher values of $p$ with appropriate coefficients. Discrete Fourier transforms are obtained as

$$
\begin{aligned}
F(f, \bar{x})= & \frac{2}{\Delta x} \int_{x_{m}}^{x_{M}} A_{0}(x) \sin \left(2 \pi f_{0} x+\phi\right) w(x) \\
& \exp (-2 i \pi f x) \mathrm{d} x .
\end{aligned}
$$

Analytical solution of equation (3) is given in the Appendix (Eq. (A.5)) for $f=f_{0}$. The modulus of $F\left(f_{0}, \bar{x}\right)$ defines the Fourier amplitude $A_{F}(\bar{x})=\left|F\left(f_{0}, \bar{x}\right)\right| / c_{0}$. For finite $\lambda$ and $f_{0} \gg \lambda$, equation (A.4) holds, yielding

$$
\begin{aligned}
A_{F}(\bar{x})= & A_{0}(\bar{x}) c_{0}^{-1} \frac{\sinh (\lambda \Delta x / 2)}{\lambda \Delta x / 2} \\
& \times \sum_{n=0}^{p}(-1)^{n} c_{n} \frac{(\lambda \Delta x / 2)^{2}}{(\lambda \Delta x / 2)^{2}+\pi^{2} n^{2}} .
\end{aligned}
$$

$A_{F}(\bar{x})$ can also be obtained by numerical resolution of equation (3), where $A_{0}(\bar{x})$ is either given by equation (2) or by experimental data of reference [9]. Available frequencies are bounded by the Raleigh frequency $\left(f_{\min }=1 / \Delta x\right)$ and by the Nyquist frequency $\left(f_{\max }=1 / 2 \delta x\right.$, for data sampled at evenly spaced $\delta x$ values). Accordingly, $\Delta x$ is kept above $1 / f_{0}$, and $\delta x$ is always small enough to ensure that $f_{\max }$ is much higher than $f_{0}[19]$.

\section{Results and discussion}

The Fourier analysis displayed in Figure 1 shows that the largest (smallest) secondary lobes and the smallest (largest) peak width are obtained for the flat (Blackman)

${ }^{1}$ At the lowest temperature $(2 \mathrm{~K})$ and highest field (55 T), i.e. lowest $u_{0} T_{m} \beta_{x}$ values explored in reference [9], this approximation overestimates the amplitude of the considered $\beta$ component by less than $3 \%$. Accordingly, the relevant reported mass plots are linear (see also Fig. 4a). 

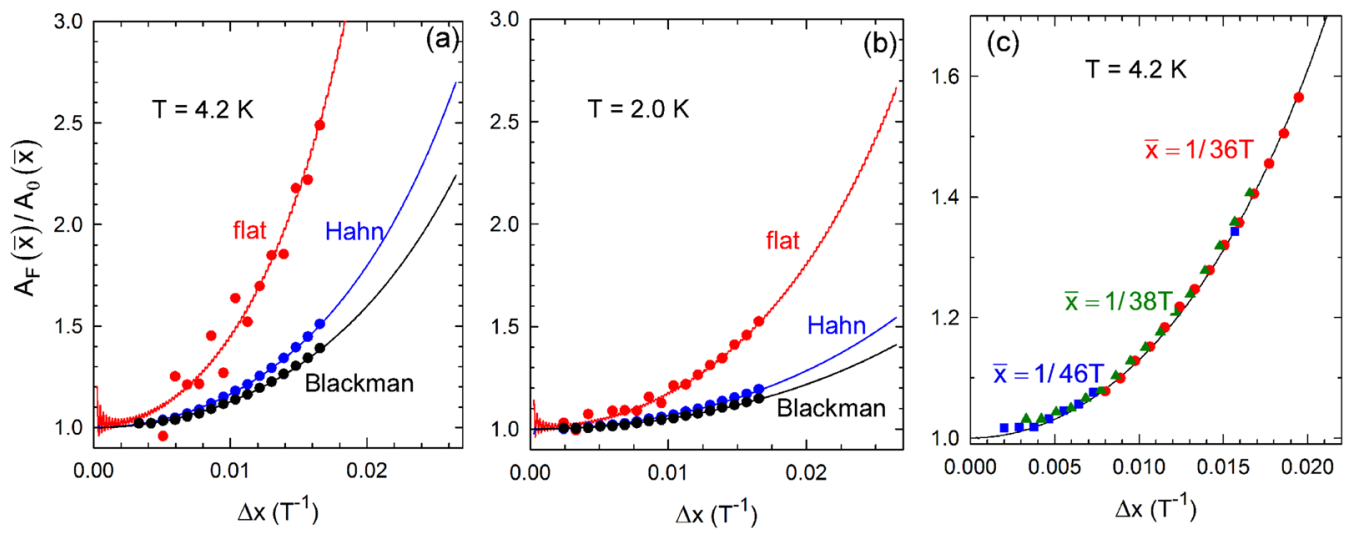

Fig. 2. Fourier amplitude $A_{F}(\bar{x})$ relevant to the $\beta$ component of the organic metal $\theta-(\mathrm{ET}){ }_{4} \mathrm{ZnBr}_{4}\left(\mathrm{C}_{6} \mathrm{H}_{4} \mathrm{Cl}_{2}\right)$ at $(\mathrm{a}) 4.2 \mathrm{~K}(\lambda=305 \mathrm{~T})$ and (b) $2.0 \mathrm{~K}(\lambda=194 \mathrm{~T})$, normalized to the oscillation amplitude predicted by the Lifshitz-Kosevich formula $A_{0}(\bar{x})$ as a function of the inverse field window $\Delta x$ for flat, Hahn and Blackman windows, at $\bar{x}=1 / 38 \mathrm{~T}$ and (c) for Blackman window at various $\bar{x}$ values. Solid symbols are deduced from experimental data reported in Ref. [9]. Solid lines are obtained from numerical resolutions of equation (3).

window, while the Hahn window provides intermediate behaviour, as widely reported [14-18].

Discrepancy between amplitude $A_{F}(\bar{x})$ deduced from the Fourier analysis within a finite field range $1 / x_{M}$ to $1 / x_{m}$ and the actual Fourier amplitude $A_{0}(\bar{x})$ given by equation (2) can be evaluated through the ratio $A_{F}(\bar{x}) / A_{0}(\bar{x})$, which should be equal to 1 . According to the data in Figure 2, a strong increase of this ratio is observed as $\Delta x$ increases. Furthermore, for a given window width $\Delta x$, it increases as $\lambda$ increases, e.g. by increasing the temperature, while as the mean magnetic field $(1 / \bar{x})$ decreases, it grows staying on the same curve, as reported in Figure 2c. The most dramatic effect is observed for the flat window, indicating that smooth windowing is necessary to get amplitudes as reliable as possible since, more specifically, $A_{F}(\bar{x}) / A_{0}(\bar{x})$ grows as $A_{F}(\bar{x}) / A_{0}(\bar{x}) \simeq$ $\sinh (\lambda \Delta x / 2) /(\lambda \Delta x / 2)$ in this case.

In line with equation (4), the ratio $A(\bar{x}) / A_{0}(\bar{x})$ depends only on the product $\lambda \Delta x$ for a given window type. Hence, strictly speaking, the Fourier analysis yields reliable amplitudes for finite $\Delta x$ in the case of fieldindependent signal $(\lambda=0)$ only. Unfavourably, moderate oscillations of the Fourier amplitude is however observed for small $\Delta x$, in particular for the flat window. It can be checked that these oscillations are periodic in $\Delta x$, their frequency being just $f_{0}$, in agreement with equation (A.5). This feature brings us to consider the influence of the quantum oscillation frequency on the data. As reported in Figure 3 , the Fourier amplitude $A_{F}(\bar{x})$ is dominated by the monotonous term of equation (A.5), yielding equations (A.4) and (4), in the case of large-enough frequency and $\Delta x$. In contrast, large oscillations of both the Fourier amplitude and the frequency of the Fourier peaks (which is no more equal to $f_{0}$ in this case) are observed for low frequencies.

This effect is relevant in the case of slow magnetic oscillations, such as superconducting iron-based chalcogenides [3-6] and in semimetal $\mathrm{BaNiS}_{2}$ [20], where frequencies as low as $37 \mathrm{~T}$ are reported. Also, slow frequency phenomena are present in magnetoresistance oscillations of organic conductors [21] or high- $\mathrm{T}_{c}$ superconductors [22] due to the mixing of two close frequencies, the amplitude of which is still in debate [21]. In these cases, Fourier amplitudes can depend strongly on the windowing process.

In addition, whereas only the envelope of $A_{F}(\bar{x})$, i.e. $A_{0}(\bar{x})$, is relevant for the Fourier amplitude at high $\Delta x$, Onsager phase-dependent data are observed in Figure 3 for low frequencies. In short, $\Delta x$ must be both small enough to avoid the amplitude overestimation predicted by equation (A.5) and large enough to avoid the undulations reported in Figure 3 in this case. As a consequence, reliable data can hardly been deduced from the Fourier analysis of low-frequency quantum oscillations.

Since $\lambda$ depends on temperature, the discrepancy between the actual and Fourier amplitudes for large $\Delta x$ depends on temperature as well. This may lead to a significant error on the effective mass deduced from temperature dependence of the amplitude (the so-called mass plot), as evidenced in Figure 4a, and hence on the determination of the scattering rate through Dingle plots as well. As reported in Figure 4b and c, underestimation of $m_{\beta}$ by about $30 \%$ is obtained for a flat window at $\bar{x}=1 / 32 \mathrm{~T}^{-1}$ for $\Delta x=0.026 \mathrm{~T}^{-1}$ (i.e. in the field range $23-56 \mathrm{~T})$. About $50 \%$ would be reached at $\bar{x}=1 / 56 \mathrm{~T}^{-1}$ for the same $\Delta x$ value (field range within 32 and $193 \mathrm{~T}$ ). Smaller although significant errors are obtained for Hahn (not shown) and Blackman windows, e.g. 15 and $13 \%$, respectively, for $\bar{x}=1 / 32 \mathrm{~T}^{-1}$ and $\Delta x=0.026 \mathrm{~T}^{-1}$.

\section{Conclusion}

Amplitude of field-dependent quantum oscillations deduced from the Fourier analysis is overestimated even though it is widely used, as reported in the literature. Analytical formulas accounting for this phenomenon are discussed in this manuscript. Most dramatic effects are observed for steep field-dependent amplitudes 

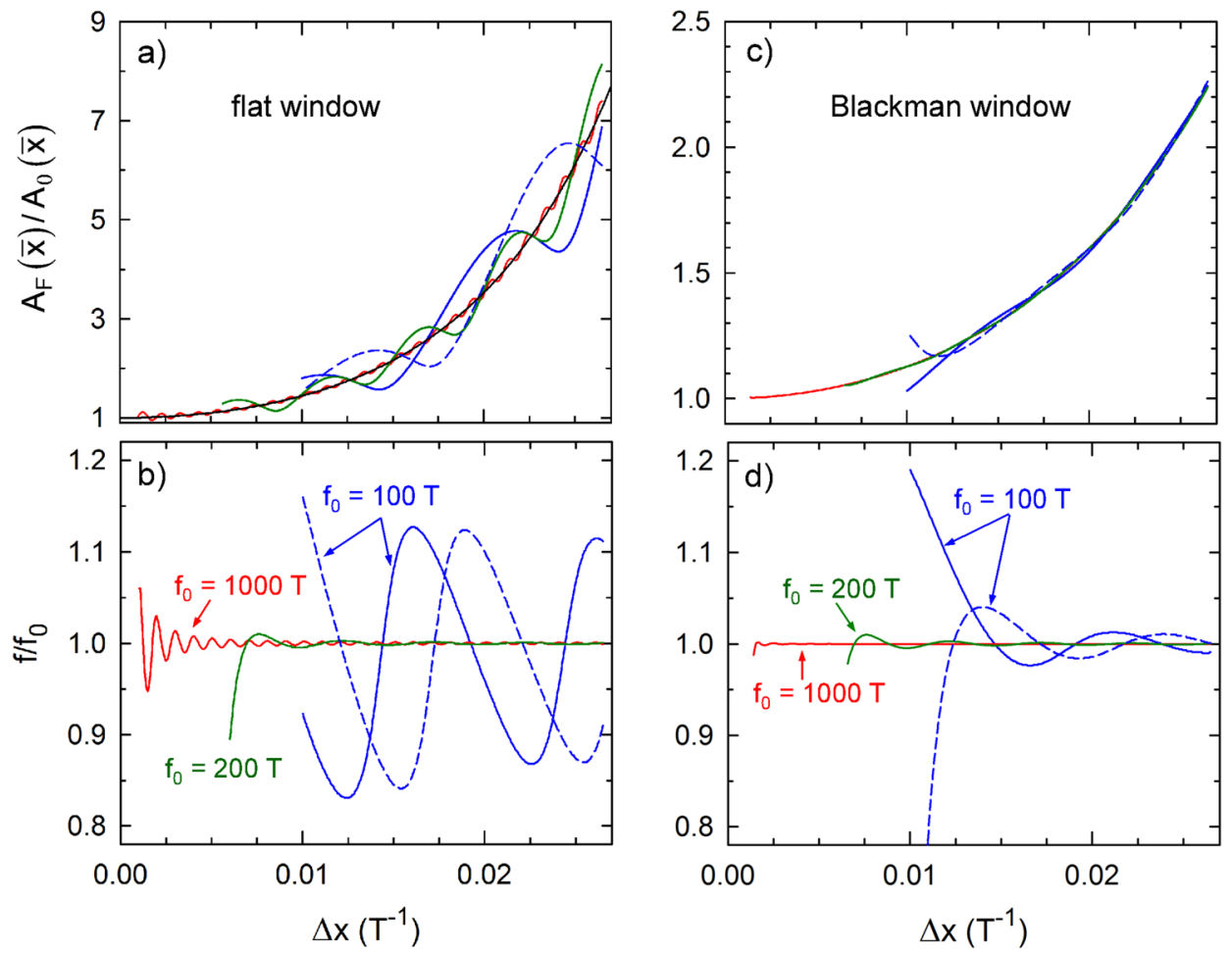

Fig. 3. Inverse field window width $(\Delta x)$ dependence of (a), (c) Fourier amplitude and (b), (d) frequency for (a), (b) flat and (c), (d) Blackman window for various oscillation frequencies $f_{0}$ and $\lambda=305 \mathrm{~T}$. The Onsager phase is $\phi=0$ and $\phi=\pi$ for solid and dashed lines, respectively. Black solid line in (a) stands for equation (4).
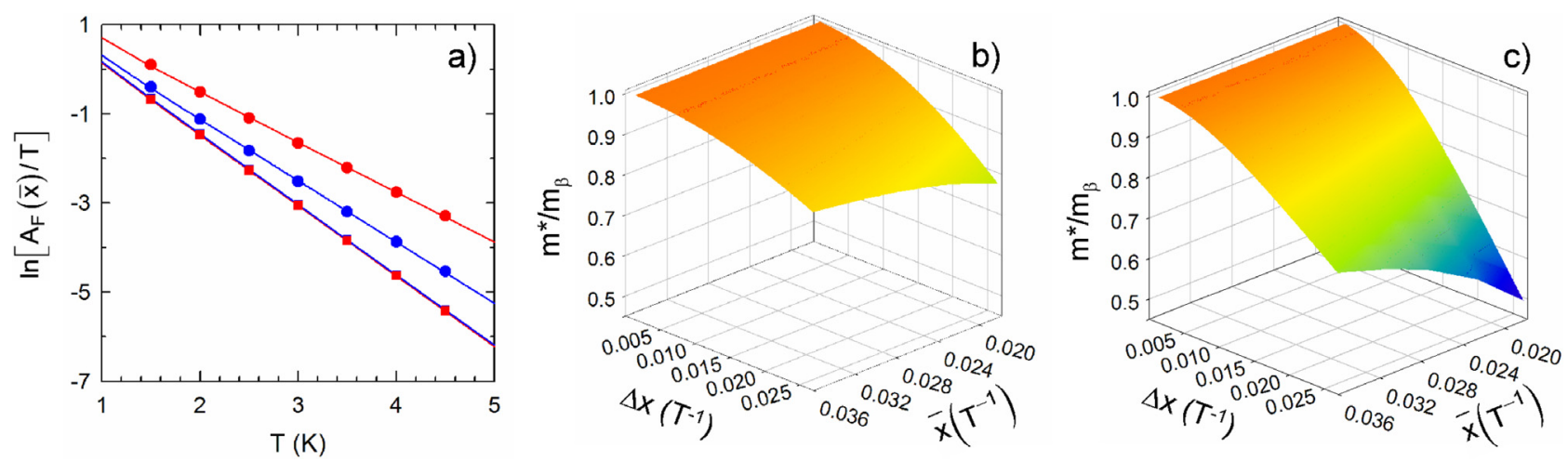

Fig. 4. (a) Mass plots relevant to the $\beta$ component of the organic metal $\theta-(\mathrm{ET})_{4} \mathrm{ZnBr}_{4}\left(\mathrm{C}_{6} \mathrm{H}_{4} \mathrm{Cl}_{2}\right)$, with effective mass $m_{\beta}=3.44$, deduced from the Fourier analysis for $\bar{x}=1 / 32 \mathrm{~T}$, in the temperature range $1.5-4.5 \mathrm{~K}$. Blue and red symbols are data obtained with Blackman and flat windows, respectively. Solid squares and circles are data for $\Delta x=0.00093 \mathrm{~T}^{-1}$ and $0.0265 \mathrm{~T}^{-1}$, respectively. Solid lines are best fits to the Lifshitz-Kosevich formula. For large $\Delta x$, both the Fourier amplitude increases and the slope decreases yielding underestimated effective mass. Such fittings yield data of Figure $4 \mathrm{~b}$ and $\mathrm{c}$, where the effective mass is plotted versus the inverse field window width $(\Delta x)$ and mean inverse field value $(\bar{x})$ for (b) Blackman and (c) flat window. At high field (i.e. small $\bar{x}$ ) and large field window width $(\Delta x)$, strong underestimation of the effective mass is obtained.

determined using flat windows with large width. Nevertheless, acceptable discrepancy with actual amplitude is obtained with Blackman window of moderate width for high-enough frequencies. In contrast, oscillations with low frequencies such as that observed in iron-based chalcogenides superconductors must be considered with care since $\Delta x$ must be both small enough to avoid overestimated amplitude and large enough to avoid spurious effects observed coming close to the inverse of the Raleigh frequency.

Work in Toulouse was supported by the European Magnetic Field Laboratory (EMFL). D. Vignolles, R.B. Lyubovskii, L. Drigo, G.V. Shilov, F. Duc, E.I. Zhilyaeva, R.N. Lyubovskaya and E. Canadell, as coauthors of reference [9], on which are based the data of Figures 1 and 2, are acknowledged. 


\section{Author contribution statement}

A. Audouard developed the idea of analysing the amplitudes of the quantum oscillations extracted from Fourier algorithms, in order to estimate their influence on the physical parameters. J.-Y. Fortin helped in writing the different formulas used in this analysis.

\section{Appendix: Analytical expression of the Fourier transforms}

In general, we can write the window function $w(x)=$ $\sum_{n \geq 0}^{p} c_{n} \cos [2 \pi n(x-\bar{x}) / \Delta x]$, where $p=0,1,2$ stands for a flat, Hahn and Blackman window, respectively, and the condition $\sum_{n=0}^{p} c_{n}=1$. These coefficients are given numerically by $\left\{c_{0}=1\right\}_{\text {Flat }},\left\{c_{0}=0.5, c_{1}=0.5\right\}_{\text {Hahn }}$ and $\left\{c_{0}=0.42, c_{1}=0.5, c_{2}=0.08\right\}_{\text {Blackman }}$. Equations (2) and (3) lead to

$$
\begin{aligned}
& F\left(f_{0}, \bar{x}\right)=\frac{1}{\Delta x} \sum_{n=0}^{p} c_{n} \sum_{\varepsilon= \pm 1} \\
& \times \int_{x_{m}}^{x_{M}} A_{0}(x) \sin \left(2 \pi f_{0} x+\phi\right) \mathrm{e}^{-2 i \pi f_{0} x-2 i \pi n \varepsilon(x-\bar{x}) / \Delta x} \mathrm{~d} x
\end{aligned}
$$

for $f=f_{0}, \quad x=\bar{x}=\left(x_{M}+x_{m}\right) / 2 \quad$ and $\quad A_{0}(x)=a_{0} \mathrm{e}^{-\lambda x}$. Writing $F\left(f_{0}, \bar{x}\right)=\sum_{n} c_{n} \sum_{\varepsilon= \pm 1} F_{n \varepsilon}$ in equation (A.1), one can compute individually $F_{n \varepsilon}$, which leads after integration to

$$
\begin{aligned}
F_{n \varepsilon}= & \frac{2 a_{0} \mathrm{e}^{2 i \pi n \varepsilon \bar{x} / \Delta x+i \phi}}{i \Delta x} \\
& \times\left[\mathrm{e}^{-\lambda_{n \varepsilon} \bar{x}} \frac{\sinh \left(\lambda_{n \varepsilon} \Delta x / 2\right)}{\lambda_{n \varepsilon}}-\mathrm{e}^{-\Lambda_{n \varepsilon} \bar{x}} \frac{\sinh \left(\Lambda_{n \varepsilon} \Delta x / 2\right)}{\Lambda_{n \varepsilon}}\right],
\end{aligned}
$$

where we have defined $\lambda_{n \varepsilon}=\lambda+2 i \pi n \varepsilon / \Delta x$ and $\Lambda_{n \varepsilon}=$ $\lambda+4 i \pi f_{0}+2 i \pi n \varepsilon / \Delta x$. This expression does not depend on $\phi$ up to a global sign, for the values $\phi=0, \pi$. Assuming $f_{0} \gg \lambda$, only the first term in bracket will contribute to $F_{n \varepsilon}$. Since $\sinh \left(\lambda_{n \varepsilon} \Delta x / 2\right)=(-1)^{n} \sinh (\lambda \Delta x / 2)$, one obtains

$$
F_{n \varepsilon} \simeq \frac{2 a_{0} \mathrm{e}^{-\lambda \bar{x}+i \phi}}{i \Delta x}(-1)^{n} \frac{\sinh (\lambda \Delta x / 2)}{\lambda+2 i \pi n \varepsilon / \Delta x} .
$$

After summing over $\varepsilon$, the Fourier transform is finally equal to

$$
\begin{aligned}
& F\left(f_{0}, \bar{x}\right) \simeq \\
& \quad-i A_{0}(\bar{x}) \mathrm{e}^{i \phi} \frac{\sinh (\lambda \Delta x / 2)}{\lambda \Delta x / 2} \sum_{n=0}^{p}(-1)^{n} c_{n} \frac{(\lambda \Delta x / 2)^{2}}{(\lambda \Delta x / 2)^{2}+\pi^{2} n^{2}} .
\end{aligned}
$$

The exact formula is obtained by incorporating the contribution from the second term of equation (A.2), involving $\Lambda_{n \varepsilon}$ and which induces oscillations as a function of $\bar{x}$ and $\Delta x$, with frequency $f_{0}$

$$
\begin{gathered}
F\left(f_{0}, \bar{x}\right)=-i A_{0}(\bar{x}) \mathrm{e}^{i \phi} \sum_{n \geq 0}(-1)^{n} c_{n} \\
{\left[\frac{\sinh (\lambda \Delta x / 2)}{\lambda \Delta x / 2} \frac{(\lambda \Delta x / 2)^{2}}{(\lambda \Delta x / 2)^{2}+\pi^{2} n^{2}}-\mathrm{e}^{-4 i \pi f_{0} \bar{x}}\right.} \\
{\left[\cos \left(2 \pi f_{0} \Delta x\right) \sinh (\lambda \Delta x / 2)\right.} \\
\left.+i \sin \left(2 \pi f_{0} \Delta x\right) \cosh (\lambda \Delta x / 2)\right] \\
\left.\frac{\left(\lambda+4 i \pi f_{0}\right) \Delta x / 2}{\left\{\left(\lambda+4 i \pi f_{0}\right) \Delta x / 2\right\}^{2}+\pi^{2} n^{2}}\right]
\end{gathered}
$$

As $\Delta x$ goes to zero for finite $\lambda$ in equation (A.4), it yields the Fourier amplitude $\left|F\left(f_{0}, \bar{x}\right)\right| \simeq A_{0}(\bar{x}) c_{0}$. As a result, one defines $A_{F}(\bar{x})=\left|F\left(f_{0}, \bar{x}\right)\right| / c_{0}$ in order to normalize the function with respect to $A_{0}(\bar{x})$ in this limit. While $\sinh (\lambda \Delta x / 2) /(\lambda \Delta x / 2)$ goes to 1 as $\Delta x$ goes to zero, the other contributions in equation (A.5), which involve oscillatory terms periodic in $\Delta x$ with the frequency $f_{0}$, grow simultaneously. They are responsible for the oscillatory behaviour reported in Figures 2 and 3. In particular, in this limit, if we take into account all the contributions in equation (A.5), one obtains

$$
\lim _{\Delta x \rightarrow 0} F\left(f_{0}, \bar{x}\right) \simeq 2 c_{0} A_{0}(\bar{x}) \mathrm{e}^{i \phi-2 i \pi f_{0} \bar{x}} \sin \left(2 \pi f_{0} \bar{x}\right) .
$$

Furthermore, as discussed in Section $2, A_{F}(\bar{x}) / A_{0}(\bar{x})$ depends only on $\lambda$ at a given $\Delta x$.

\section{References}

1. D. Shoenberg, Magnetic Oscillations in Metals (Cambridge University Press, Cambridge, UK, 1984)

2. Y. Onuki, R. Settai, Low Temp. Phys. 38, 89 (2012)

3. T. Terashima, N. Kikugawa, A. Kiswandhi, E.-S. Choi, J.S. Brooks, S. Kasahara, T. Watashige, H. Ikeda, T. Shibauchi, Y. Matsuda, T. Wolf, A.E.B. Böhmer, F. Hardy, C. Meingast, H.V. Löhneysen, M.-T. Suzuki, R. Arita, S. Uji, Phys. Rev. B 90, 144517 (2014)

4. M.D. Watson, T.K. Kim, A.A. Haghighirad, N.R. Davies, A. McCollam, A. Narayanan, S.F. Blake, Y.L. Chen, S. Ghannadzadeh, A.J. Schofield, M. Hoesch, C. Meingast, T. Wolf, A.I. Coldea, Phys. Rev. B 91, 155106 (2015)

5. M.D. Watson, T. Yamashita, S. Kasahara, W. Knafo, M. Nardone, J. Béard, F. Hardy, A. McCollam, A. Narayanan, S.F. Blake, T. Wolf, A.A. Haghighirad, C. Meingast, A.J. Schofield, H.V. Löhneysen, Y. Matsuda, A.I. Coldea, T. Shibauchi, Phys. Rev. Lett. 115, 027006 (2015)

6. A. Audouard, F. Duc, L. Drigo, P. Toulemonde, S. Karlsson, P. Strobel, A. Sulpice, EPL 109, 27003 (2015)

7. S. Uji, J.S. Brooks, Springer Series Material Science (Springer, New York City, 2008), Vol. 110, p. 89

8. A. Audouard, J.-Y. Fortin, C. R. Phys. 14, 15 (2013)

9. A. Audouard, J.-Y. Fortin, D. Vignolles, R.B. Lyubovskii, L. Drigo, G.V. Shilov, F. Duc, E.I. Zhilyaeva, R.N. Lyubovskaya, E. Canadell, J. Phys. Condens. Matter 27, 315601 (2015)

10. A.B. Pippard, Proc. R. Soc. Lond. A270, 1 (1962) 
11. A. Audouard, J.-Y. Fortin, D. Vignolles, R.B. Lyubovskii, L. Drigo, F. Duc, G.V. Shilov, G. Ballon, E.I. Zhilyaeva, R.N. Lyubovskaya, E. Canadell, EPL 97, 57003 (2012)

12. I.M. Lifschitz, A.M. Kosevich, Sov. Phys. JETP 2, 636 (1956)

13. L.M. Falicov, H. Stachowiak, Phys. Rev. 147, 505 (1966)

14. A.S. Filler, J. Opt. Soc. Am. 54, 762 (1964)

15. J. Allen, IEEE Trans. Acoust. Speech Signal Process. ASSP25, 235 (1977)

16. F.J. Harris, Proc. IEEE 66, 51 (1978)

17. F.S. Acton, Numerical Methods That Work (The Mathematical Association of America, Washington D.C., 1990)

18. D.A. Naylor, M.K. Tahic, J. Opt. Soc. Am. A 24, 3644 (2007)
19. L. Drigo, F. Durantel, A. Audouard, G. Ballon, Eur. Phys. J. Appl. Phys. 52, 10401 (2010)

20. Y. Klein, M. Casula, D. Santos-Cottin, A. Audouard, D. Vignolles, G. Fve, V. Freulon, B. Plaçais, M. Verseils, H. Yang, L. Paulatto, A. Gauzzi, Phys. Rev. B 97, 075140 (2018)

21. P.D. Grigoriev, T.I. Mogilyuk, Phys. Rev. B 95, 195130 (2017)

22. P.D. Grigoriev, T. Ziman, Phys. Rev. B 96, 165110 (1017)

Open Access This article is distributed under the terms of the Creative Commons Attribution License https://creativecom mons.org/licenses/by/4.0 which permits unrestricted use, distribution, and reproduction in any medium, provided the original author(s) and source are credited.

Cite this article as: Alain Audouard, Jean-Yves Fortin, Does Fourier analysis yield reliable amplitudes of quantum oscillations? Eur. Phys. J. Appl. Phys. 83, 30201 (2018) 